\title{
RESEARCH
}

Open Access

\section{The Latin American Spanish version of the Face-Name Associative Memory Exam is sensitive to cognitive and pathological changes in preclinical autosomal dominant Alzheimer's disease}

Clara Vila-Castelar ${ }^{1 \dagger}$, Nathalia Muñoz ${ }^{1 \dagger}$, Kathryn V. Papp ${ }^{2,3}$, Rebecca E. Amariglio ${ }^{2,3}$, Ana Baena ${ }^{4}$, Edmarie Guzmán-Vélez ${ }^{1}$, Yamile Bocanegra ${ }^{4}$, Justin S. Sanchez ${ }^{3}$, Eric M. Reiman ${ }^{5}$, Keith A. Johnson ${ }^{2,6}$, Reisa A. Sperling ${ }^{2,3,7}$, Francisco Lopera ${ }^{4}$, Dorene M. Rentz ${ }^{2,3}$ and Yakeel T. Quiroz ${ }^{1,3,4^{*}}$

\begin{abstract}
Background: To determine whether performance on the Latin American Spanish version of the Face-Name Associative Memory Exam (LAS-FNAME) can differentiate between cognitively intact carriers of an autosomal dominant Alzheimer's disease mutation (E280A) in Presenilin-1, who are genetically determined to develop earlyonset dementia, from matched non-carriers. We also sought to examine whether LAS-FNAME performance is associated with amyloid- $\beta$ and regional tau burden in mutation carriers.

Methods: A total of 35 cognitively intact mutation carriers (age range 26-41), 19 symptomatic carriers, and 48 matched non-carriers (age range 27-44) completed a neuropsychological assessment including the LAS-FNAME. A subset of participants (31 carriers [12 symptomatic] and 35 non-carriers) traveled from Colombia to Boston to undergo positron emission tomography (PET) using Pittsburgh compound B to measure mean cortical amyloid- $\beta$ and flortaucipir for regional tau. ANOVA analyses and Spearman correlations were used to examine group differences and relationships among LAS-FNAME performance and amyloid- $\beta$ and tau accumulation.

Results: Compared to non-carriers, cognitively intact mutation carriers had lower scores on the LAS-FNAME Total Scores $(p=.040)$. Across all carriers (including symptomatic carriers), higher levels of amyloid- $\beta(r=-.436, p=.018)$ and regional tau in the entorhinal $(r=-.394, p=.031)$ and inferior temporal cortex $(r=-.563, p=.001)$ were associated with lower LAS-FNAME Total Scores.

(Continued on next page)
\end{abstract}

\footnotetext{
*Correspondence: yquiroz@mgh.harvard.edu

${ }^{\dagger}$ Clara Vila-Castelar and Nathalia Muñoz contributed equally to this work.

'Department of Psychiatry, Massachusetts General Hospital, Harvard Medical School, Boston, MA, USA

${ }^{3}$ Department of Neurology, Massachusetts General Hospital, Harvard Medical School, Boston, MA, USA

Full list of author information is available at the end of the article
}

(c) The Author(s). 2020 Open Access This article is licensed under a Creative Commons Attribution 4.0 International License, which permits use, sharing, adaptation, distribution and reproduction in any medium or format, as long as you give appropriate credit to the original author(s) and the source, provide a link to the Creative Commons licence, and indicate if changes were made. The images or other third party material in this article are included in the article's Creative Commons licence, unless indicated otherwise in a credit line to the material. If material is not included in the article's Creative Commons licence and your intended use is not permitted by statutory regulation or exceeds the permitted use, you will need to obtain permission directly from the copyright holder. To view a copy of this licence, visit http://creativecommons.org/licenses/by/4.0/. The Creative Commons Public Domain Dedication waiver (http://creativecommons.org/publicdomain/zero/1.0/) applies to the data made available in this article, unless otherwise stated in a credit line to the data. 
(Continued from previous page)

Conclusions: Performance on the LAS-FNAME differentiated between cognitively intact mutation carriers from noncarriers and was associated with greater amyloid and tau burden when examining all carriers. Findings suggest that the LAS-FNAME is sensitive to early clinical and pathological changes and can potentially help track disease progression in Spanish-speaking individuals.

Keywords: Alzheimer's disease, Autosomal dominant Alzheimer's disease, Neuropsychology, Associative memory, Imaging, PET

\section{Introduction}

Alzheimer's disease (AD) affects 6 million individuals in the USA [1]. Latinos have a higher rate of AD than nonHispanic whites [2], which is expected to rise rapidly due to population aging [3]. Yet, there is an underrepresentation of Latinos in clinical trials to prevent $\mathrm{AD}$. There are growing efforts to promote recruitment of under-represented groups into research studies. Therefore, there is a need to develop neuropsychological measures to detect AD-pathology in Spanish-speaking individuals and track cognitive changes along the $\mathrm{AD}$ trajectory [4].

We validated the Latin American Spanish-Face-Name Associative Memory Exam (LAS-FNAME) [5], an adaptation of the Face-Name Associative Memory Exam (FNAME) to assess Spanish-speaking individuals. The FNAME is a highly demanding, associative memory test, which tracks memory changes along the AD-spectrum [6-8] that has been shown to be correlated with $A \beta$ accumulation in clinically normal older adults $[7,9]$ and adults with subjective cognitive decline (SCD) [10]. Recent evidence also showed that performance on a computerized version of the FNAME was associated with cerebrospinal fluid levels of amyloid, phosphorylated tau, and total tau in clinically normal and mild cognitive impairment (MCI) [11]. Importantly, the FNAME engages memory network regions, including the hippocampus, and default mode network regions that are affected early in the disease process [12]. Thus, the FNAME is a challenging and multimodal tool that detects early associative memory changes related to AD.

We leveraged data from the largest kindred with autosomal dominant AD (ADAD) due to a mutation (E280A) in Presenilin-1 (PSEN1), who are virtually destined to develop early-onset AD. We examined whether performance on the LAS-FNAME can distinguish cognitively intact individuals at genetic risk to develop dementia later in life, and whether LAS-FNAME performance is related to $A \beta$ and tau accumulation in the brain. We hypothesized that cognitively intact PSEN1 mutation carriers would perform worse on the LAS-FNAME than non-carriers and that greater levels of $A \beta$ and tau accumulation would be associated with worse LAS-FNAME performance. We also explored performance on subscales of the LAS-FNAME and its association to AD-related pathology in PSEN1 mutation carriers.

\section{Methods \\ Study design and participants}

One hundred and thirteen individuals from the Colombia-Boston biomarker study (COLBOS) were included. Participants lived in or near Medellín, Colombia, and were recruited from the Alzheimer's Prevention Initiative registry. Participants were under the age of 50 and had a parent with the autosomal dominant AD due to E280A mutation in PSEN1. The clinical staging of this kindred is characterized by an onset of MCI [13] at a median age of 44 years old ( $95 \%$ C.I. $=43,45$ years) and dementia at 49 years old (95\% C.I. $=49,50$ years) [14].

Cognitively intact participants had to show no cognitive impairment on a standard cognitive battery, including a Mini-Mental State Examination (MMSE) [15] score $\geq 26$ and a Functional Assessment Staging test (FAST) [16] $=1$ (indicating no cognitive impairment or subjective cognitive concerns). Thirty-five cognitively intact participants were PSEN1 mutation carriers (mean age $32.14 \pm 3.73$ years; $51 \%$ females), and forty-eight were age-matched non-carrier family members (mean age $34.43 \pm 4.32$ years; $56 \%$ females) (Table 1). Symptomatic carriers $(n=19)$ were defined as having a FAST score of 2 , indicating the presence of subjective cognitive concerns $(n=11$, mean age $38.36 \pm 4.92)$, or a FAST score of 3 , indicating the presence of MCI $(n=8$, mean age $43.75 \pm 2.91$ ). Mutation carriers and non-carriers were age-matched. Potential participants were screened and excluded in advance for the presence of neurological or chronic psychiatric disorders, and eligibility to undergo MRI. Participants and investigators were blind to genetic status.

\section{Procedures}

Ethics approval was obtained from the University of Antioquia (Colombia) Ethics Committee and the Partners Human Research Institutional Review Board. Clinical and cognitive evaluations (including the LAS- 
Table 1 Demographic, cognitive, and clinical characteristics of the sample

\begin{tabular}{|c|c|c|c|c|c|}
\hline & Non-carriers $(\boldsymbol{n}=48)$ & Cognitively intact carriers $(\boldsymbol{n}=35)$ & $p^{a}$ & Symptomatic carriers $(\boldsymbol{n}=19)$ & $p^{b}$ \\
\hline & $M(S D)$ & $M(S D)$ & & $M(S D)$ & \\
\hline Demographic and clinical variables & & & & & \\
\hline Age (years) & $34.43(4.32)$ & $32.14(3.73)$ & $.018^{*}$ & $40.63(4.92)$ & .557 \\
\hline Education (years) & $10.45(4.19)$ & $10.72(3.68)$ & .769 & $7.16(3.84)$ & .259 \\
\hline MMSE & $28.94(.88)$ & $28.67(.93)$ & .199 & $25.63(3.17)$ & $.001^{*}$ \\
\hline GDS & $1.00(.00)$ & $1.03(.00)$ & 1.000 & $2.84(3.35)$ & .798 \\
\hline Cognitive variables & & & & & \\
\hline CERAD Word List Learning & $20.63(2.98)$ & $20.28(3.54)$ & .799 & $13.84(4.39)$ & $.007^{*}$ \\
\hline CERAD Word List Delayed Recall & $7.67(1.26)$ & $7.31(1.58)$ & .534 & $3.74(2.64)$ & $.002^{*}$ \\
\hline CERAD Word List Recognition & $9.71(.58)$ & $9.81(.47)$ & .486 & $7.95(2.20)$ & $.034^{*}$ \\
\hline CERAD Constructional Praxis-Copy & $10.41(.89)$ & $10.25(.91)$ & .346 & $9.53(1.12)$ & $.030^{*}$ \\
\hline CERAD Constructional Praxis-Delayed Recall & $9.57(1.70)$ & $9.72(1.52)$ & .744 & $4.63(3.48)$ & $.036^{*}$ \\
\hline Phonemic Fluency (FAS) & $32.41(7.33)$ & $34.53(7.89)$ & .212 & $31.47(10.38)$ & .868 \\
\hline Semantic Fluency (Animals) & $20.37(4.01)$ & $21.31(4.20)$ & .233 & $18.74(3.97)$ & .888 \\
\hline Boston Naming Test (BNT-15) & $13.82(.95)$ & $13.33(1.24)$ & .055 & $12.95(1.71)$ & $.023^{*}$ \\
\hline PET variables^^ & & & & & \\
\hline Amyloid (Aß) (DVR) & $1.11(0.04)$ & $1.45(.24)$ & $.000^{*}$ & $1.93(.30)$ & $.000^{*}$ \\
\hline Entorhinal cortex tau (SUVR) & $1.05(0.21)$ & $1.34(.42)$ & .054 & $2.10(.70)$ & $.000^{*}$ \\
\hline Inferior temporal cortex tau (SUVR) & $1.18(0.11)$ & $1.23(.16)$ & .108 & $1.72(.46)$ & $.001^{*}$ \\
\hline
\end{tabular}

Note: Symptomatic carriers include participants with FAST scores of 2 and 3 indicating presence of SCD and $\mathrm{MCl}$, respectively

Abbreviations: $M$ mean, SD standard deviation, FAST Functional Assessment Staging test, MMSE Mini Mental State Exam, GDS Geriatric Depression Scale, CERAD

Consortium to Establish a Registry for Alzheimer's Disease, DVR distribution volume ratio, SUVR standardized uptake value ratio

$\wedge$ From subset participants with PET data. All PET values were partial volume corrected (PVC)

${ }^{a} p$ value calculated for Mann-Whitney test for cognitively intact PSEN1 mutation carriers versus non-carriers

${ }^{\mathrm{b}} p$ value calculated for Mann-Whitney test for all PSEN1 mutation carriers (i.e., cognitively intact, SCD, and MCI) versus non-carriers

${ }^{*} p<0.05$. Group differences significant at the 0.05 level (2-tailed)

FNAME task) were administered in Spanish by psychometrists and neuropsychologists at the Grupo de Neurociencias de Antioquia within 2 months of brain imaging. One hundred and two individuals completed these evaluations, including 35 cognitively intact mutation carriers (age range 26-41), 48 matched non-carriers (aged 27$44)$, and 19 symptomatic carriers (13 SCD and $6 \mathrm{MCI}$ ). A subset of participants $(n=66), 31$ carriers (6 SCD, 6 $\mathrm{MCI})$ and 35 non-carriers, traveled to Boston to complete magnetic resonance imaging (MRI) and positron emission tomography (PET) imaging. One participant could not complete the 11C Pittsburgh compound $\mathrm{B}$ (PiB) PET scan due to radiotracer production failure.

Cognitive testing included the Consortium to Establish a Registry for Alzheimer's Disease neuropsychological battery (CERAD) word list and constructional praxis, Boston Naming Test (BNT-15), MMSE, Geriatric Depression Scale [17] and FAST [16].

\section{LAS-FNAME task description}

Detailed description of this task can be found elsewhere [5]. In brief, during the initial learning, participants are shown a succession of 12 faces with a name written below on a computer screen. Participants are asked to recognize the target face between two distractors (Immediate Recognition-Face), provide the initial letter of the name associated with the learned face (Immediate Recall-Letter), and finally, identify the name that was paired with each face among three choices (Immediate Recognition-Name). Items correctly identified for each condition (Face, Letter, Name) are summed for an Immediate Memory Total score (/36).

Following a 25-min delay, participants are asked to identify again the learned face between two distractors (Delayed Recognition-Face), recall the initial letter of the name associated with each face (Delayed RecallLetter), and select the name per face among three choices (Delayed Recognition-Name). Items correctly identified for each condition (Face, Letter, Name) are summed for a Delayed Memory Total score (/36). Immediate and Delayed Memory total scores are summed to produce a LAS-FNAME Total Score (/72).

\section{Brain imaging}

Participants underwent tau and amyloid PET imaging at Massachusetts General Hospital, Boston. As previously 
reported [18], PiB PET was acquired with a $8.5-15 \mathrm{mCi}$ bolus injection followed immediately by a 60 -min dynamic acquisition in 69 frames $(12 \times 15 \mathrm{~s}, 57 \times 60 \mathrm{~s})$. [F18] Flortaucipir (FTP) was acquired between 80 and $100 \mathrm{~min}$ after a $9.0-11.0 \mathrm{mCi}$ bolus injection in 4 separate 5 -min frames.

11C PiB PET data were expressed as the distribution volume ratio (DVR) with cerebellar gray matter as reference tissue; regional time-activity curves were used to compute regional DVRs for each region of interest (ROI) using the Logan graphical method applied to data obtained between 40 and $60 \mathrm{~min}$ after injection [19]. $11 \mathrm{C} \mathrm{PiB}$ retention was assessed using a large cortical ROI aggregate that included frontal, lateral temporal, and retrosplenial cortices [20].

[F18] FTP-specific binding was expressed in FreeSurfer ROIs as the standardized uptake value ratio (SUVR) to cerebellar gray matter [21]. We selected FTP binding on bilateral entorhinal and inferior temporal cortices (EC and IT) as these regions are both crucial for associative memory, and early sites of tau accumulation in ADAD [18]. PET data were corrected for partial volume effects using the geometric transfer matrix method [22]. For whole-brain analyses of PiB and FTP, DVR and SUVR images were normalized to standard space and smoothed with an 8-mm Gaussian kernel to account for individual anatomic differences [23].

\section{Statistical analyses}

Statistical analyses were performed using SPSS [24]. Mann-Whitney tests were conducted to compare demographic and clinical characteristics between PSEN1 mutation carriers and non-carriers. An analysis of variance was used to compare LAS-FNAME Total Score, between cognitively intact PSEN1 mutation carriers and non-carriers. We then conducted the same analysis including all PSEN1 mutation carriers (i.e., cognitively intact, SCD, and $\mathrm{MCI}$ ) and matched noncarriers to examine whether the LAS-FNAME was sensitive to capture cognitive changes along the disease trajectory. Effect sizes (i.e., Cohen's $d$ ) and models adjusting for age and years of education are reported. Spearman's correlations examined the relationship between LAS-FNAME Total Score, mean cortical A $\beta$ and regional tau accumulation in the EC and IT in cognitively intact PSEN1 mutation carriers to examine how performance on the LAS-FNAME was associated with early markers of AD-pathology accumulation. We then conducted the same analysis across all PSEN1 mutation carriers to examine how performance on the LASFNAME relates to accumulation of pathology through preclinical stages. No correction for multiple comparisons was employed due to small sample size.
Exploratory analyses compared performance on the LAS-FNAME subscales (i.e., Immediate Memory Total and Delayed Memory Total) and conditions (i.e., Face Recognition, Letter Recall, and Name Recognition) between PSEN1 mutation carriers and non-carriers. Lastly, we carried out exploratory whole-brain analyses examining the relationship between $A \beta$ and tau burden and LAS-FNAME Total Score in PSEN1 mutation carriers. Regions were $p<0.05$ after cluster-wise false discovery rate correction for multiple comparisons (minimum cluster extent $k=100 \mathrm{~mm}^{2}$ ).

\section{Results}

Demographic, clinical, and cognitive measures in carriers and non-carriers

Demographic, cognitive, and clinical data are presented in Tables 1 and 2. Cognitively intact PSEN1 mutation carriers were significantly younger than non-carriers $(p=.018)$. There were no differences in educational level or percentage of females. Cognitively intact PSEN1 mutation carriers had slightly lower BNT-15 scores $(p=$ .055) compared to non-carriers. No other differences were found among cognitive measures. As expected, symptomatic carriers were older, had lower MMSE scores, and performed worse across most cognitive measures than non-carriers.

\section{LAS-FNAME performance in PSEN1 mutation carriers and non-carriers}

Cognitively intact PSEN1 mutations carriers had significantly lower scores on the LAS-FNAME Total Score $(p=.040)$ and Immediate Memory $(p=.037)$ and showed a trend towards lower scores on Delayed Memory ( $p=$ .059) than non-carriers (Table 3 and Fig. 1). When adjusting for age and years of education, cognitively intact carriers showed a trend towards lower scores on the Immediate Memory $(F(1,83)=3.92, p=.051)$, Delayed Memory $(F(1,83)=2.92, p=.092)$, and LAS-FNAME Total Score $(F(1,83)=3.64, p=.060)$.

Exploratory analyses showed that within Immediate Memory conditions, cognitively intact PSEN1 mutation carriers had significantly lower scores on Name Recognition $(p=.006)$ than non-carriers. Within Delayed Memory conditions, cognitively intact PSEN1 mutation carriers performed significantly lower on Letter Recall $(p=.043)$ than non-carriers.

Performance on the LAS-FNAME across all PSEN1 mutation carriers showed that symptomatic carriers had significantly lower LAS-FNAME Total Score $(p=.001)$, Immediate Memory $(p=.001)$, and Delayed Memory $(p=.001)$ than non-carriers (Table 4$)$. When adjusting for age and years of education across all mutation carriers, carriers exhibited significantly lower Immediate Memory $(F(1,111)=11.83, p=.001)$, Delayed Memory 
Table 2 Demographic and clinical characteristics of subset participants with PET data

\begin{tabular}{llll}
\hline & $\begin{array}{l}\text { Non-carriers }(\boldsymbol{n}=35) \\
\text { M (SD) }\end{array}$ & Cognitively intact carriers $(\boldsymbol{n}=19)$ & Symptomatic carriers $(\boldsymbol{n}=12)$ \\
\hline $\begin{array}{l}\text { Demographic and clinical variables } \\
\text { Age (years) }\end{array}$ & $34.69(4.84)$ & $33.95(5.08)$ & $42.25(3.41)$ \\
Education (years) & $11.03(4.16)$ & $11.21(3.15)$ & $5.75(3.14)$ \\
MMSE & $28.97(.92)$ & $28.68(.88)$ & $25.08(3.23)$ \\
GDS & $.94(1.49)$ & $.63(1.46)$ & $2.75(3.22)$ \\
Cognitive variables & & & $12.50(3.87)$ \\
CERAD Word List Learning & $20.91(3.15)$ & $20.05(2.99)$ & $2.92(2.39)$ \\
CERAD Word List Delayed Recall & $7.69(1.25)$ & $7.05(1.96)$ & $7.33(2.27)$ \\
CERAD Word List Recognition & $9.74(.56)$ & $9.84(.37)$ & $9.50(1.31)$ \\
CERAD CP- Copy & $10.40(1.03)$ & $10.58(.61)$ & $3.83(3.43)$ \\
CERAD CP- Delayed Recall & $9.51(1.58)$ & $10.05(1.58)$ & $29.17(8.40)$ \\
Phonemic Fluency (FAS) & $32.54(7.69)$ & $36.74(7.96)$ & $18.25(3.93)$ \\
Semantic Fluency (Animals) & $20.00(3.66)$ & $21.68(4.70)$ & $13.00(1.76)$ \\
Boston Naming Test (BNT-15) & $13.86(1.00)$ & $13.58(1.17)$ &
\end{tabular}

Note: Symptomatic carriers include participants with FAST scores of 2 and 3 indicating presence of SCD concerns and MCl, respectively Abbreviations: $M$ mean, SD standard deviation, FAST Functional Assessment Staging test, MMSE Mini Mental State Exam, GDS Geriatric Depression Scale, CERAD Consortium to Establish a Registry for Alzheimer's Disease, $C P$ constructional praxis

$(F(1,111)=10.67, p=.001)$, and LAS-FNAME Total Score $(F(1,111)=12.13, p=.001)$.

Exploratory analyses showed that within conditions, symptomatic carriers performed significantly lower on Immediate Memory-Letter Recall $(p=.024)$ and Name Recognition $(p=.000)$, as well as Delayed Memory-Letter Recall $(p=.001)$ and Name Recognition ( $p=$ .011) than non-carriers. Symptomatic PSEN1 mutation carriers showed a trend towards lower scores on Delayed Memory-Face Recognition $(p=.058)$, while Immediate
Memory-Face Recognition was not significantly different than in non-carriers $(p=.114)$.

\section{Age and LAS-FNAME performance in PSEN1 mutation carriers}

Age was negatively correlated with performance on the LAS-FNAME, wherein older cognitively intact PSEN1 mutation carriers have lower LAS-FNAME Total $(r=-.163 ; p=.350)$, Immediate Memory $(r=$ $-.190 ; p=.275)$, and Delayed Memory score $(r=$

Table 3 Differences in LAS-FNAME performance between cognitively intact PSEN1 mutation carriers and non-carriers

\begin{tabular}{|c|c|c|c|c|c|c|}
\hline \multirow[b]{2}{*}{ LAS-FNAME } & \multirow{2}{*}{$\begin{array}{l}\text { Non-carriers }(\boldsymbol{n}=48) \\
M(S D)\end{array}$} & \multicolumn{5}{|c|}{ Cognitively intact carriers $(\boldsymbol{n}=35)$} \\
\hline & & $M(S D)$ & $d f$ & $F$ & $p$ value $^{a}$ & $d$ \\
\hline \multicolumn{7}{|l|}{ Immediate Memory } \\
\hline Face Recognition & $11.71(.79)$ & $11.60(.74)$ & 1,83 & .40 & .530 & .14 \\
\hline Letter Recall & $4.88(2.60)$ & $4.20(2.29)$ & 1,83 & 1.50 & .225 & .28 \\
\hline Name Recognition & $9.83(1.70)$ & $8.54(2.45)$ & 1,83 & 8.09 & $.006^{*}$ & .62 \\
\hline \multicolumn{7}{|l|}{ Delayed Memory } \\
\hline Face Recognition & $11.81(.53)$ & $11.80(2.45)$ & 1,83 & .10 & .919 & .01 \\
\hline Letter Recall & $6.06(2.98)$ & $4.80(2.42)$ & 1,83 & 4.24 & $.043^{*}$ & .47 \\
\hline Name Recognition & $9.27(2.39)$ & $8.46(2.62)$ & 1,83 & 2.17 & .145 & .32 \\
\hline \multicolumn{7}{|l|}{ Scale Scores } \\
\hline IM Total & $26.42(4.19)$ & $24.34(4.66)$ & 1,83 & 4.51 & $.037^{*}$ & .47 \\
\hline DM Total & $27.14(4.88)$ & $25.06(4.93)$ & 1,83 & 3.67 & .059 & .42 \\
\hline LAS-FNAME Total & $53.56(8.76)$ & $49.40(9.26)$ & 1,83 & 4.35 & $.040^{*}$ & .46 \\
\hline
\end{tabular}

Abbreviations: $M$ mean, SD standard deviation, IM Immediate Memory, DM Delayed Memory, LAS-FNAME Total IM Total + DM Total, df degrees of freedom, F Fratio, $d$ Cohen's $d$ effect size

${ }^{a} p$ value calculated for ANOVA tests for cognitively intact PSEN1 mutation carriers versus non-carriers

${ }^{*} p<0.05$ 


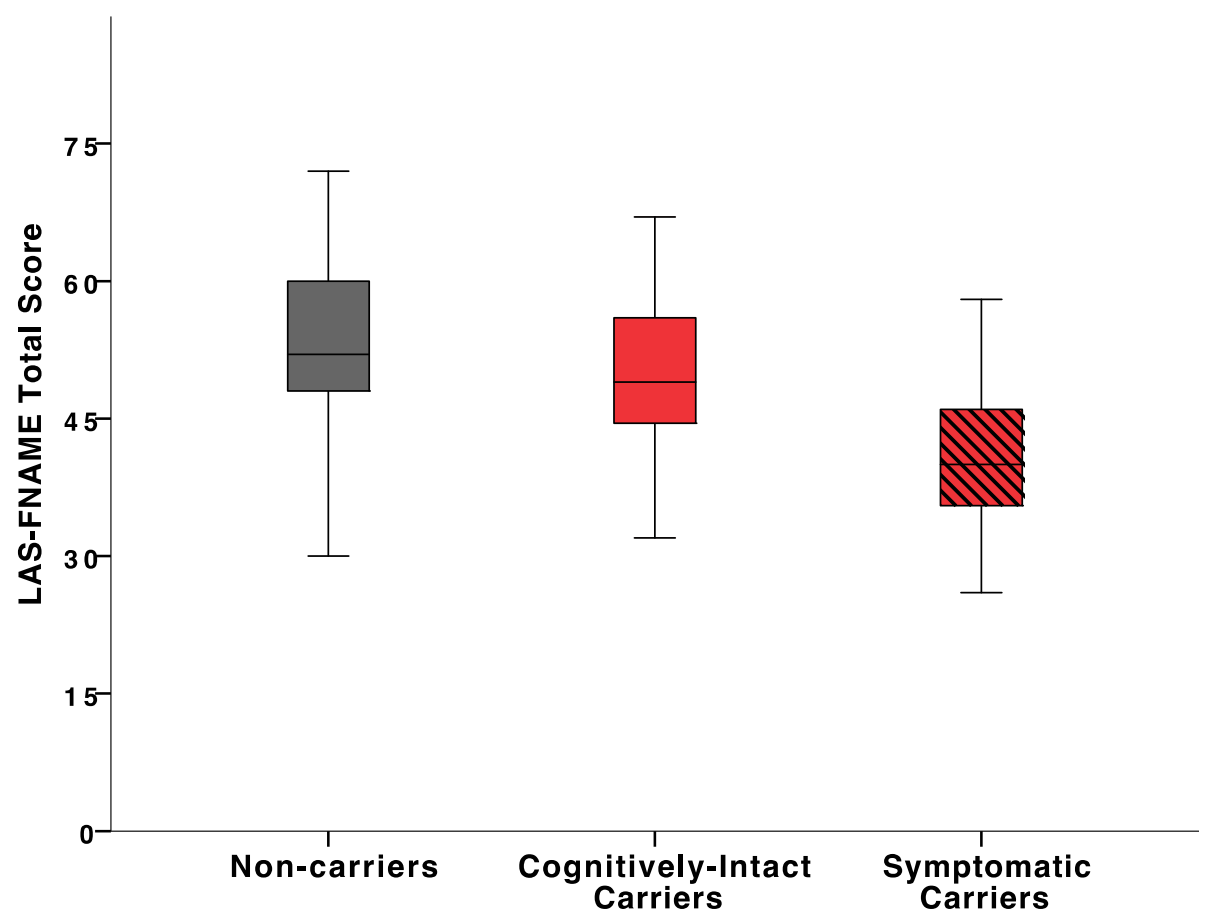

Fig. 1 Note. Performance on the LAS-FNAME Total Score in PSEN1 mutation carriers and non-carriers. Cognitively intact PSEN1 mutations carriers had significantly lower LAS-FNAME Total Score $(p=.040)$ than non-carriers. Across all PSEN1 mutation carriers (i.e., cognitively intact, subjective cognitive decline, and mild cognitive impairment), carriers had significantly lower LAS-FNAME Total Score $(p=.001)$ than non-carriers

Table 4 Differences in LAS-FNAME performance between symptomatic PSEN1 mutation carriers and non-carriers

\begin{tabular}{|c|c|c|c|c|c|c|}
\hline \multirow[b]{2}{*}{ LAS-FNAME } & \multirow{2}{*}{$\begin{array}{l}\text { Non-carriers }(\boldsymbol{n}=48) \\
M(S D)\end{array}$} & \multicolumn{5}{|c|}{ Symptomatic carriers $(\boldsymbol{n}=19)$} \\
\hline & & $M(S D)$ & $d f$ & $F$ & $p$ value $^{a}$ & $d$ \\
\hline \multicolumn{7}{|l|}{ Immediate Memory } \\
\hline Face Recognition & $11.71(.79)$ & $10.84(1.71)$ & 1,111 & 2.53 & .114 & .38 \\
\hline Letter Recall & $4.88(2.60)$ & $2.84(1.89)$ & 1,111 & 5.25 & $.024^{*}$ & .49 \\
\hline Name Recognition & $9.83(1.70)$ & $7.00(2.21)$ & 1,111 & 18.61 & $.000^{*}$ & .86 \\
\hline \multicolumn{7}{|l|}{ Delayed Memory } \\
\hline Face Recognition & $11.81(.53)$ & $10.58(1.80)$ & 1,111 & 3.68 & .058 & .47 \\
\hline Letter Recall & $6.06(2.98)$ & $3.11(2.05)$ & 1,111 & 11.12 & $.001^{*}$ & .70 \\
\hline Name Recognition & $9.27(2.39)$ & $7.11(1.91)$ & 1,111 & 6.71 & $.011^{*}$ & .52 \\
\hline \multicolumn{7}{|l|}{ Scale Scores } \\
\hline IM Total & $26.42(4.19)$ & $20.68(4.40)$ & 1,111 & 12.58 & $.001^{*}$ & .75 \\
\hline DM Total & $27.14(4.88)$ & $20.79(4.43)$ & 1,111 & 11.45 & $.001^{*}$ & .71 \\
\hline LAS-FNAME Total & $53.56(8.76)$ & $41.47(8.32)$ & 1,111 & 12.77 & $.001^{*}$ & .76 \\
\hline
\end{tabular}

Note: Symptomatic carriers include participants with FAST scores of 2 and 3 indicating presence of subjective cognitive concerns and mild cognitive impairment, respectively

Abbreviations: $M$ mean, SD standard deviation, IM Immediate Memory, DM Delayed Memory, LAS-FNAME Total IM Total + DM Total, df degrees of freedom, F Fratio, $d$ Cohen's d effect size

${ }^{a} p$ value calculated for ANOVA tests for all PSEN1 mutation carriers (i.e., cognitively intact, subjective cognitive concerns, and mild cognitive impairment) versus non-carriers

${ }^{*} p<0.05$ 
$-.109 ; p=.531)$; however, these associations did not reach significance (Fig. 2a). In contrast, when examining the relationship between age and LAS-FNAME scores across all carriers, greater age was significantly associated with lower performance on the LASFNAME Total Score $(r=-.364, p=.006)$ and all its subscales (Immediate Memory: $r=-.328, p=.014$; Delayed Memory: $r=-.356, p=.008$ ).
$A \beta$ burden and LAS-FNAME performance in PSEN1 mutation carriers

Cognitively intact PSEN1 mutation carriers had significantly higher $\mathrm{A} \beta$ burden than non-carriers $(p=.000)$. $\mathrm{A} \beta$ burden was significantly higher across all PSEN1 mutation carriers than in non-carriers $(p=.000)$ (Table 1).

There was no association between performance on the LAS-FNAME and A $\beta$ burden in cognitively intact PSEN1

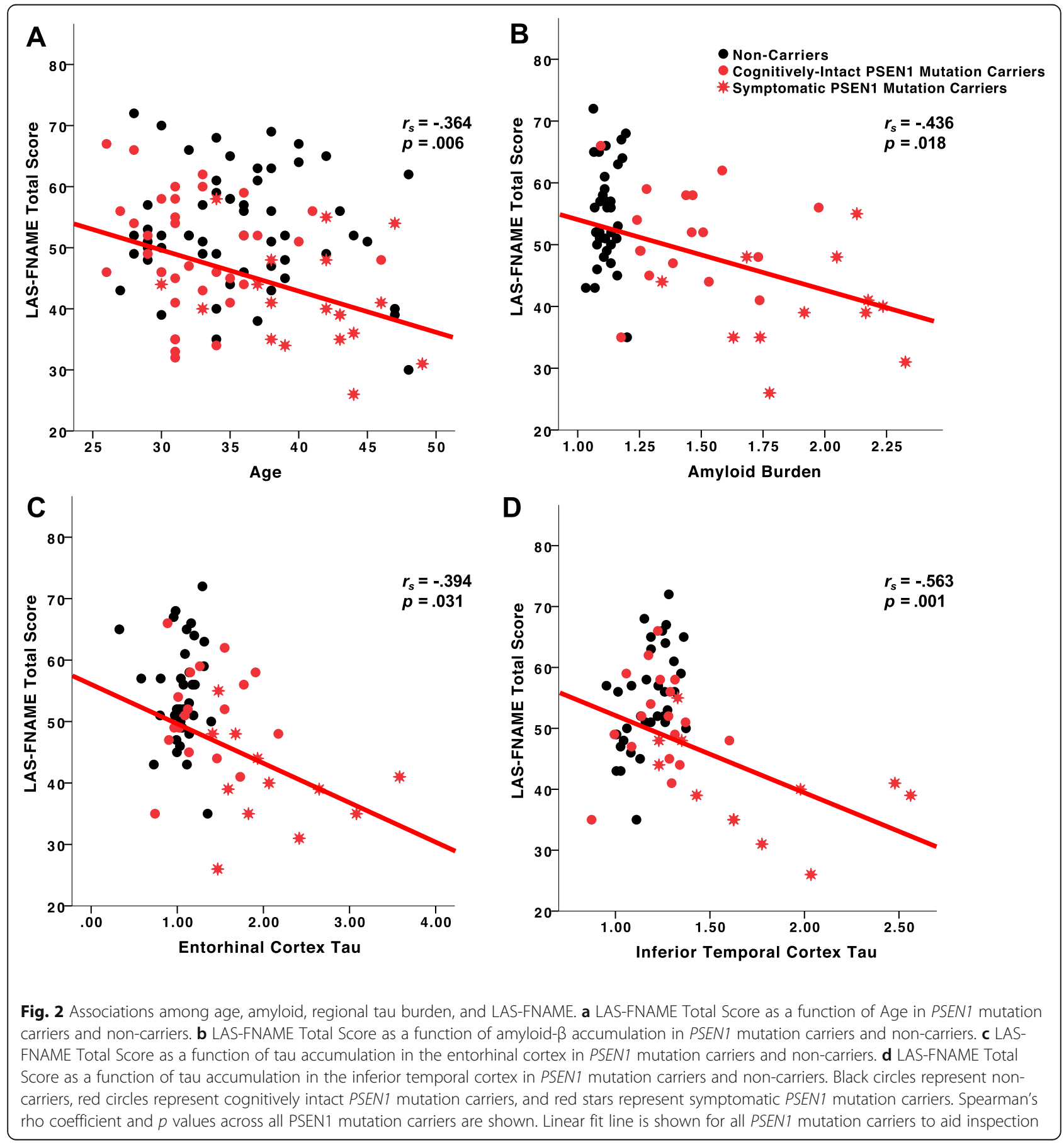


mutation carriers (Immediate Memory: $r=-.111, p=.683$; Delayed Memory: $r=-.077, p=.777$; Total Score: $r=$ $-.016, p=.952)$. We then examined the association between $\mathrm{A} \beta$ burden and performance on the LAS-FNAME across all PSEN1 mutation carriers and found that greater A $\beta$ burden was significantly associated with lower LASFNAME Total Score $(r=-.436, p=.018)$ (Fig. 2b), Immediate Memory $(r=-.438, p=.017)$, and Delayed Memory $(r=-.417, p=.024)$ scores.

\section{Tau and LAS-FNAME performance in PSEN1 mutation carriers}

Cognitively intact PSEN1 mutation carriers showed a trend towards higher levels of EC tau $(p=.054)$ than non-carriers, while there was no difference in IT tau $(p=.108)$. Compared to non-carriers, both EC and IT tau were significantly higher across all carriers (EC tau: $p=.000$; IT tau: $p=.001$ ) (Table 1$)$.

Among cognitively intact PSEN1 mutation carriers, performance on the LAS-FNAME was not associated with EC tau (Immediate Memory: $r=-.085, p=.746$; Delayed Memory: $r=.303, p=.237$; Total Score: $r=.236, p=.362$ ). When examining these associations across all PSEN1 mutation carriers, we found that greater EC tau was associated with lower LAS-FNAME Total Score $(r=$ $-.394, p=.031$ ) (Fig. 2c) and Immediate Memory scores $(r=-.435, p=.016)$, while Delayed Memory scores showed a trend towards significance $(r=-.350, p=.058)$.

Finally, we examined the association between IT tau and LAS-FNAME scores and found that among cognitively intact PSEN1 mutation carriers, LAS-FNAME scores were not associated with IT tau (Immediate Memory: $r=-.188, p=.469$; Delayed Memory: $r=.086$, $p=.742$; Total Score: $r=-.082, p=.754)$. In contrast, across all PSEN1 mutation carriers, greater IT tau was significantly associated with lower LAS-FNAME Total Score $(r=-.563, p=.001)$ (Fig. 2d), Immediate Memory Total $(r=-.570, p=.001)$ and Delayed Memory Total scores $(r=-.528, p=.003)$.

\section{Whole-brain analyses of the relationship between pathology burden and LAS-FNAME}

We examined the relationship between LAS-FNAME Total Score and $A \beta$ and tau burden in the whole brain within PSEN1 carriers. Whole-brain analyses showed no significant relationships between $A \beta$ burden and LASFNAME Total Score after FDR correction for multiple comparisons. In contrast, whole-brain analyses between tau burden and LAS-FNAME Total Score showed a pattern consistent with findings from regions selected a priori, wherein LAS-FNAME Total Score was related to higher tau burden in inferior and medial temporal regions (i.e., EC and IT), and parietal regions (i.e., precuneus and inferior parietal) (Fig. 3).

\section{Discussion}

The FNAME is an associative memory test sensitive to early changes in $A D$, which has been associated with $A \beta$ and CSF tau accumulation in clinically normal [7, 9], SCD [10], and MCI [11]. Our aim was to explore whether the LAS-FNAME can help identify individuals at increased risk for developing AD by differentiating between cognitively intact PSEN1 mutation carriers and non-carriers. Further, we explored the relationship among performance on the LAS-FNAME and in vivo markers of $A \beta$ and tau accumulation in PSEN1 mutation carriers who underwent PET imaging.

Our findings suggest that the LAS-FNAME can distinguish between cognitively intact PSEN1 mutation carriers and non-carriers. Cognitively intact carriers had lower LAS-FNAME Total Scores despite being significantly younger than non-carriers and showed a trend towards lower LAS-FNAME Total Scores when adjusting for age and years of education. These findings are remarkable as we defined cognitively intact carriers using stringent criteria, namely absence of objectively defined cognitive impairment and subjective cognitive concerns. Notably, no other cognitive measure was able to distinguish between cognitively intact carriers and noncarriers. Differences in LAS-FNAME scores were even greater when examining across all PSEN1 mutation carriers by including symptomatic carriers (i.e., subjective cognitive decline and mild cognitive impairment) who were further along the disease trajectory. Thus, our findings highlight the potential of the LAS-FNAME as a sensitive cognitive measure to capture subtle memory changes that appear in the early, preclinical stages of $\mathrm{AD}$.

In autosomal dominant $\mathrm{AD}$, age is commonly used as a proxy of disease progression, because as carriers age, they get closer to their age of estimated symptom onset. We found that age and performance on the LASFNAME were not significantly associated in cognitively intact PSEN1 mutation carriers. However, when examining this relationship across all carriers, older age was associated with lower performance on the LAS-FNAME, suggesting that the LAS-FNAME is sensitive to progression of associative memory difficulties from preclinical to mildly symptomatic stages of AD. These findings are also notable in that our sample is remarkably younger than previous studies, highlighting the potential applicability of the FNAME across age ranges.

Our findings suggest that $A \beta$ burden was not associated with LAS-FNAME scores in cognitively intact PSEN1 mutation carriers whereas higher $\mathrm{A} \beta$ burden was associated with lower LAS-FNAME Total Scores when including mildly symptomatic PSEN1 mutation carriers (i.e., carriers with subjective cognitive decline and $\mathrm{MCI}$ ). Previous research has shown that the FNAME was 


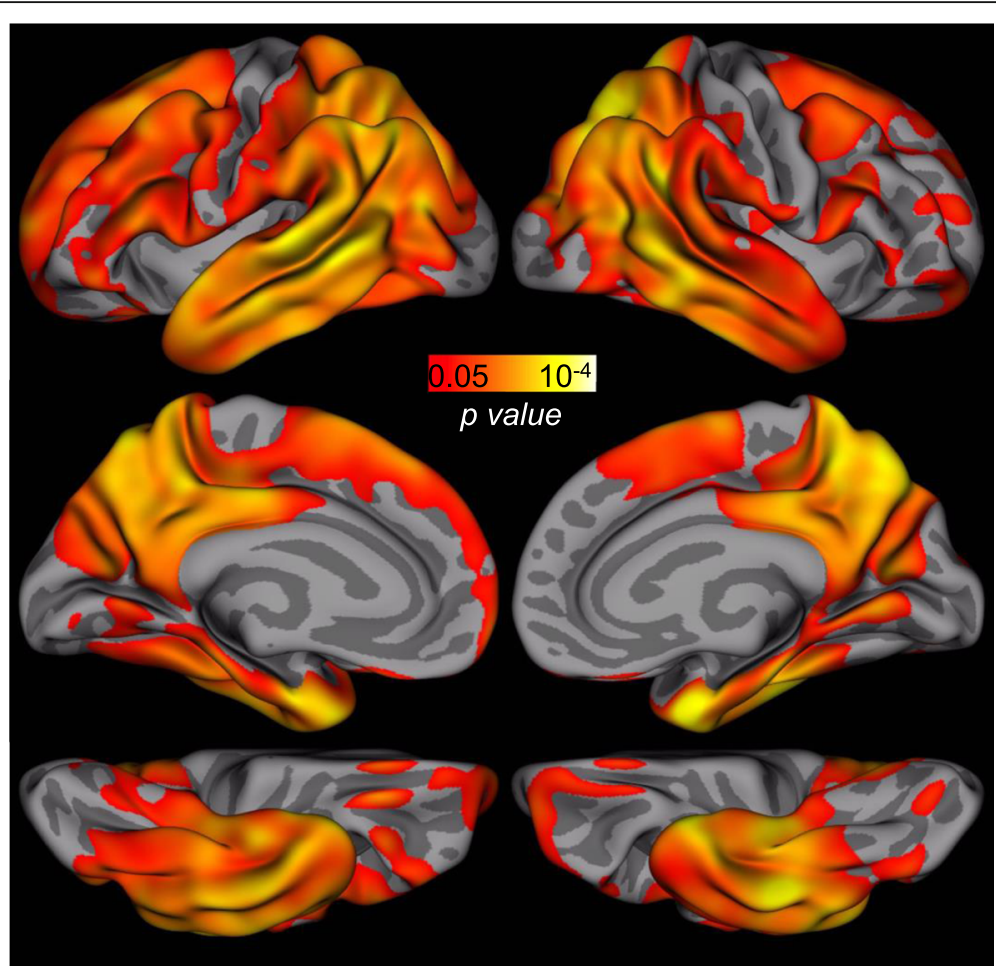

Fig. 3 Whole-brain relationships between tau PET measures and LAS-FNAME Total Score. Note. Whole-brain relationships between tau PET measures and LAS-FNAME Total Score within PSEN1 E280A carriers. Results are displayed as $-\log _{10}(p), p<0.05$ after cluster-wise false discovery rate (FDR) correction for multiple comparisons, minimum cluster size $=100 \mathrm{~mm}^{2}$. No relationships between PiB PET and LAS-FNAME Total Score were significant after FDR correction

associated with $\mathrm{A} \beta$ burden in clinically normal older adults $[7,9]$. However, our findings do not support such association in cognitively intact mutation carriers. This discrepancy is likely due to the sample's limited age range, which also leads to restricted pathology levels, as PSEN1 mutation carriers show consistently elevated levels of amyloid at preclinical stages [18]. Thus, when including carriers who are further along the disease and have greater levels of amyloid, the range of $A \beta$ burden values increased, corroborating the association between $\mathrm{A} \beta$ burden and LAS-FNAME performance.

To our knowledge, this is the first study investigating the relationship between FNAME performance and PET tau pathology. We examined regional tau accumulation in the entorhinal and inferior temporal cortices, as these regions are both crucial for associative memory [25-27] and sensitive to early disease pathology [18]. We found no association among cognitively intact PSEN1 mutation carriers. We then examined these associations among cognitively intact and symptomatic PSEN1 mutation carriers and found that greater tau accumulation in the EC and IT were associated with lower LAS-FNAME Total Score. Limited range of tau values among cognitively intact PSEN1 mutation carriers may explain the lack of association between LAS-FNAME and tau burden.
Alternatively, our findings may also suggest that higher levels of tau are required to detect an association between tau burden and LAS-FNAME, as in this kindred, tau pathology is not observed in temporal regions until close to the clinical onset. Notably, when examining these associations in mildly symptomatic individuals further along the $\mathrm{AD}$ trajectory, by including carriers with subjective cognitive concerns and mild cognitive impairment, we found that the LAS-FNAME was associated with tau burden in the entorhinal and inferior temporal cortices, early sites of tau accumulation. Moreover, whole-brain analyses confirmed that performance on the LAS-FNAME was related to higher tau burden in inferior and medial temporal regions, including entorhinal and inferior temporal cortices, as well as parietal regions such as the precuneus and inferior parietal. Overall, our findings suggest that the LAS-FNAME is sensitive to early increases in tau pathology before dementia onset.

\section{Limitations}

This study also had some limitations. First, correction for multiple comparisons was not employed due to concerns regarding relatively small sample size and power limitations. Nonetheless, this is a rare and homogeneous 
sample, as all carriers had a single mutation in PSEN1 and a well-characterized clinical and disease course. Furthermore, a relatively small sample size among participants who underwent PET imaging may have limited our ability to detect associations between LAS-FNAME performance and pathology burden among cognitively intact PSEN1 mutation carriers. Lastly, it will be important to investigate how our findings generalize to other $\mathrm{AD}$-causing mutations and the larger sporadic AD population. Future work will require longitudinal data to evaluate whether the LAS-FNAME can track cognitive changes across the AD-spectrum.

\section{Conclusions}

Performance on the LAS-FNAME was able to distinguish cognitively intact PSEN1 mutation carriers from non-carriers suggesting that the LAS-FNAME can detect early, subtle cognitive changes in individuals who will develop $\mathrm{AD}$ with virtual certainty. Furthermore, lower performance on the LAS-FNAME was associated with AD-related pathology accumulation across mildly symptomatic mutation carriers. These findings suggest that subtle changes in associative memory are evident in individuals at genetic risk for $\mathrm{AD}$ and are related to $\mathrm{AD}$ related pathology, even early in the disease process, before dementia onset. Thus, the LAS-FNAME may be a useful tool for the detection of preclinical and early $\mathrm{AD}$ in Spanish-speaking individuals. Our findings promote efforts to recruit Spanish-speaking individuals from under-represented groups in research studies and ongoing clinical trials to prevent AD.

\section{Abbreviations}

AD: Alzheimer's disease; LAS-FNAME: Latin American Spanish- Face-Name Associative Memory Exam; FNAME: Face-Name Associative Memory Exam; $A \beta$ : Amyloid-beta; SCD: Subjective cognitive decline; MCl: Mild cognitive impairment; ADAD: Autosomal dominant Alzheimer's disease;

PSEN1: Presenilin-1; MMSE: Mini-Mental State Examination; FAST: Functional Assessment Staging test; MRI: Magnetic resonance imaging; PET: Positron emission tomography; CERAD: Consortium to Establish a Registry for Alzheimer's Disease; BNT: Boston Naming Test; PiB: 11C Pittsburg Compound B; FTP: [F18] Flortaucipir; DVR: Distribution volume ratio; ROI: Region of interest; SUVR: Standardized uptake value ratio; EC: Entorhinal cortex; IT: Inferior temporal cortex

\section{Acknowledgements}

The authors thank the PSEN1 Colombian families for contributing their valuable time and effort, without which this study would not have been possible. We would also like to thank our study personnel including Alexander Navarro and Francisco Piedrahita from the Grupo de Neurociencias de Antioquia (Medellin, Colombia) and Enmanuelle PardillaDelgado, Jairo Martinez, Heirangi Torrico-Teave, Diana Munera, Joshua FoxFuller, María Alejandra Restrepo, and Arabiye Artola from the Massachusetts General Hospital (Boston, USA).

\section{Authors' contributions}

CVC, NM, KVP, REA, EMR, KAJ, RAS, FL, DMR, and YTQ contributed to study concept and design. CVC, NM, AB, EGV, YB, JSS, and YTQ contributed to data acquisition. CVC, NM, KVP, REA, EGV, YB, DMR, and YTQ contributed to analysis and interpretation of data. CVC, NM, and YTQ contributed to drafting the manuscript. CVC, NM, and JSS contributed to drafting the figures. All authors critically reviewed and approved of the manuscript.

\section{Funding}

This study was supported by the NIH National Institute of Aging (RO1AG054671 [YTQ]) and Office of the Director (DP5OD019833 [YTQ]), the Massachusetts General Hospital Executive Committee on Research (YTQ), and the Alzheimer's Association (CVC, YTQ).

\section{Availability of data and materials}

Anonymized clinical, genetic, and imaging data are available upon request, subject to an internal review by Y.T.Q. and F.L. to ensure that the participants' anonymity, confidentiality, and PSEN1 E280A carrier or noncarrier status are protected. Data requests will be considered based on a proposal review, and completion of a data sharing agreement, in accordance with the University of Antioquia and MGH institutional guidelines. Please submit data requests to Y.T.Q. (yquiroz@mgh.harvard.edu)

\section{Ethics approval and consent to participate}

Ethics approval for this study was obtained from the University of Antioquia (Colombia) Ethics Committee and the Partners Human Research Institutional Review Board. Participants provided written informed consent before enrollment into the study.

\section{Consent for publication}

Not applicable.

\section{Competing interests}

The authors declare that they have no competing interests.

\section{Author details}

'Department of Psychiatry, Massachusetts General Hospital, Harvard Medical School, Boston, MA, USA. 'Department of Neurology, Brigham and Women's Hospital, Harvard Medical School, Boston, MA, USA. ${ }^{3}$ Department of Neurology, Massachusetts General Hospital, Harvard Medical School, Boston, MA, USA. ${ }^{4}$ Grupo de Neurociencias de Antioquia, Universidad de Antioquia, Medellín, Colombia. ${ }^{5}$ Banner Alzheimer's Institute, Phoenix, AZ, USA. ${ }^{6}$ Department of Radiology, Massachusetts General Hospital, Harvard Medical School, Boston, MA, USA. 7Athinoula A. Martinos Center for Biomedical Imaging, Massachusetts General Hospital, Harvard Medical School, Charlestown, MA, USA.

Received: 11 May 2020 Accepted: 25 August 2020

Published online: 10 September 2020

\section{References}

1. Alzheimer's Association. 2019 Alzheimer's disease facts and figures. Alzheimers Dement. 2019;15(3):321-87.

2. Mayeda ER, Glymour MM, Quesenberry CP, Whitmer RA. Inequalities in dementia incidence between six racial and ethnic groups over 14 years. Alzheimers Dement. 2016;12(3):216-24.

3. Custodio N, Wheelock A, Thumala D, Slachevsky A. Dementia in Latin America: epidemiological evidence and implications for public policy. Front Aging Neurosci. 2017;9:221

4. Sperling R, Rentz DM, Johnson KA, Karlawish J, Donohue M, Salmon DP, et al. The A4 study: stopping AD before symptoms begin? Sci Transl Med. 2014;6(]):228fs13.

5. Vila-Castelar C, Papp KV, Amariglio RE, Torres VL, Baena A, Gomez D, et al. Validation of the Latin American Spanish version of the face-name associative memory exam in a Colombian Sample. Clin Neuropsychol. 2019: 1-12. https://doi.org/10.1080/13854046.2019.1690050.

6. Papp KV, Amariglio RE, Dekhtyar M, Roy K, Wigman S, Bamfo R, et al. Development of a psychometrically equivalent short form of the FaceName Associative Memory Exam for use along the early Alzheimer's disease trajectory. Clin Neuropsychol. 2014;28(5):771-85.

7. Rentz DM, Amariglio RE, Becker JA, Frey M, Olson LE, Frishe K, et al. Facename associative memory performance is related to amyloid burden in normal elderly. Neuropsychologia. 2011;49(9):2776-83.

8. Amariglio RE, Becker JA, Carmasin J, Wadsworth LP, Lorius N, Sullivan C, et al. Subjective cognitive complaints and amyloid burden in cognitively normal older individuals. Neuropsychologia. 2012;50(12):2880-6. 
9. Sperling R, Laviolette PS, O'Keefe K, O'Brien J, Rentz DM, Pihlajamaki M, et al. Amyloid deposition is associated with impaired default network function in older persons without dementia. Neuron. 2009;63(2):178-88.

10. Sanabria A, Alegret M, Rodriguez-Gomez O, Valero S, Sotolongo-Grau O, Monte-Rubio G, et al. The Spanish version of Face-Name Associative Memory Exam (S-FNAME) performance is related to amyloid burden in Subjective Cognitive Decline. Sci Rep. 2018;8(1):3828.

11. Alegret M, Muñoz N, Roberto N, Rentz DM, Valero S, Gil S, et al. A computerized version of the Short Form of the Face-Name Associative Memory Exam (FACEmemory ${ }^{\oplus}$ ) for the early detection of Alzheimer's disease. Alzheimers Res Ther. 2020;12(1):1-11.

12. Sperling R, Chua E, Cocchiarella A, Rand-Giovannetti E, Poldrack R, Schacter $\mathrm{DL}$, et al. Putting names to faces:: Successful encoding of associative memories activates the anterior hippocampal formation. Neuroimage. 2003; 20(2):1400-10.

13. Jack CR Jr, Albert MS, Knopman DS, McKhann GM, Sperling RA, Carrillo MC, et al. Introduction to the recommendations from the National Institute on Aging-Alzheimer's Association workgroups on diagnostic guidelines for Alzheimer's disease. Alzheimers Dement. 2011;7(3):257-62.

14. Acosta-Baena N, Sepulveda-Falla D, Lopera-Gómez CM, Jaramillo-Elorza MC, Moreno S, Aguirre-Acevedo DC, et al. Pre-dementia clinical stages in presenilin 1 E280A familial early-onset Alzheimer's disease: a retrospective cohort study. Lancet Neurol. 2011;10(3):213-20.

15. Folstein MF, Folstein SE, McHugh PR. "Mini-mental state": a practical method for grading the cognitive state of patients for the clinician. J Psychiatr Res. 1975;12(3):189-98.

16. Reisberg B. Functional assessment staging (FAST). Psychopharmacol Bull. 1988;24(4):653-9.

17. Yesavage JA, Brink TL, Rose TL, Lum O, Huang V, Adey M, et al. Development and validation of a geriatric depression screening scale: a preliminary report. J Psychiatr Res. 1982;17(1):37-49.

18. Quiroz YT, Sperling RA, Norton DJ, Baena A, Arboleda-Velasquez JF, Cosio $\mathrm{D}$, et al. Association between amyloid and tau accumulation in young adults with autosomal dominant Alzheimer disease. JAMA Neurol. 2018;75(5):548-56.

19. Logan J, Fowler JS, Volkow ND, Wolf AP, Dewey SL, Schlyer DJ, et al. Graphical analysis of reversible radioligand binding from time-activity measurements applied to [N-11C-methyl]-(-)-cocaine PET studies in human subjects. J Cereb Blood Flow Metab. 1990;10(5):740-7.

20. Amariglio RE, Mormino EC, Pietras AC, Marshall GA, Vannini P, Johnson KA, et al. Subjective cognitive concerns, amyloid-beta, and neurodegeneration in clinically normal elderly. Neurology. 2015;85(1):56-62.

21. Johnson KA, Schultz A, Betensky RA, Becker JA, Sepulcre J, Rentz D, et al. Tau positron emission tomographic imaging in aging and early Alzheimer disease. Ann Neurol. 2016;79(1):110-9.

22. Rousset $\mathrm{OG}, \mathrm{Ma} \mathrm{Y}$, Evans $\mathrm{AC}$. Correction for partial volume effects in PET: principle and validation. J Nucl Med. 1998;39(5):904-11.

23. Chien DT, Szardenings AK, Bahri S, Walsh JC, Mu F, Xia C, et al. Early clinica PET imaging results with the novel PHF-tau radioligand [F18]-T808. J Alzheimers Dis. 2014:38(1):171-84.

24. IBM SPSS Statistics for Windows. 25 ed. Armonk: IBM Corp; 2017.

25. Ranganath C, Cohen MX, Dam C, D'Esposito M. Inferior temporal, prefrontal, and hippocampal contributions to visual working memory maintenance and associative memory retrieval. J Neurosci. 2004;24(16):3917-25.

26. Carr VA, Bernstein JD, Favila SE, Rutt BK, Kerchner GA, Wagner AD. Individual differences in associative memory among older adults explained by hippocampal subfield structure and function. Proc Natl Acad Sci U S A. 2017;114(45):12075-80.

27. Marks SM, Lockhart SN, Baker SL, Jagust WJ. Tau and beta-amyloid are associated with medial temporal lobe structure, function, and memory encoding in Normal aging. J Neurosci. 2017;37(12):3192-201.

\section{Publisher's Note}

Springer Nature remains neutral with regard to jurisdictional claims in published maps and institutional affiliations.

Ready to submit your research? Choose BMC and benefit from:
- fast, convenient online submission
- thorough peer review by experienced researchers in your field
- rapid publication on acceptance
- support for research data, including large and complex data types
- gold Open Access which fosters wider collaboration and increased citations
- maximum visibility for your research: over 100M website views per year
At BMC, research is always in progress.
Learn more biomedcentral.com/submissions

